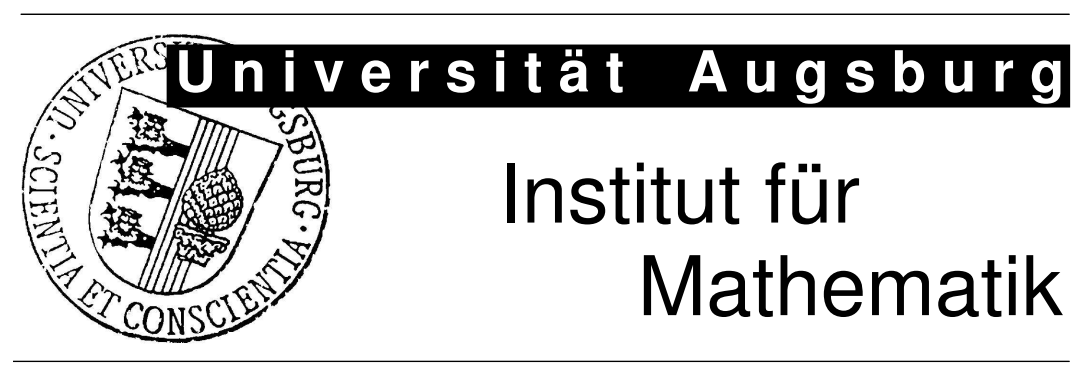

Lothar Heinrich, Malte Spiess

Berry-Esseen Bounds and Cramér-Type Large Deviations for the Volume Distribution of Poisson Cylinder Processes 


\section{Impressum:}

\section{Herausgeber:}

Institut für Mathematik

Universität Augsburg

86135 Augsburg

http://www.math.uni-augsburg.de/pages/de/forschung/preprints.shtml

\section{ViSdP:}

Lothar Heinrich

Institut für Mathematik

Universität Augsburg

86135 Augsburg

Preprint: Sämtliche Rechte verbleiben den Autoren (C) 2009 


\title{
BERRY-ESSEEN BOUNDS AND CRAMÉR-TYPE LARGE DEVIATIONS FOR THE VOLUME DISTRIBUTION OF POISSON CYLINDER PROCESSES
}

\author{
L. Heinrich ${ }^{1}$ and M. Spiess ${ }^{2}$ \\ ${ }^{1}$ Universität Augsburg, Institut für Math., Universitätsstr. 14, 86135 Augsburg, Germany \\ ${ }^{2}$ Universität Ulm, Institut für Stochastik, Helmholtzstr. 18, 86069 Ulm, Germany \\ (e-mail: heinrich@math.uni-augsburg.de and malte.spiess@uni-ulm.de)
}

\begin{abstract}
A stationary Poisson cylinder process $\Pi_{c y l}^{(d, k)}$ is composed by a stationary Poisson process of $k$-flats in $\mathbb{R}^{d}$ which are dilated by i.i.d. random compact cylinder bases taken from the corresponding orthogonal complement. We study the accuracy of normal approximation of the $d$-volume $V_{\varrho}^{(d, k)}$ of the union set of $\Pi_{c y l}^{(d, k)}$ that covers $\varrho W$ as the scaling factor $\varrho$ becomes large. Here $W$ is some fixed compact star-shaped set containing the origin as inner point. We give lower and upper bounds of the variance of $V_{\varrho}^{(d, k)}$ which exhibit long-range dependence within the union set of cylinders. Our main results are sharp estimates of the higher-order cumulants of $V_{\varrho}^{(d, k)}$ under the assumption that the $(d-k)$-volume of the typical cylinder base possesses a finite exponential moment. These estimates enable us to apply Statulevičius' celebrated 'Lemma on large deviations'.
\end{abstract}

Keywords: Random (closed) set, stationary 0 - 1-random field, volume fraction, central limit theorem, higher-order (mixed) cumulants, moment- and cumulant-generating function

MSC 2000: Primary 60 D 05, 60 F 05; Secondary 60 F 10, 60 G 55

\section{Introduction and Preliminaries}

In integral and stochastic geometry, a cylinder in $\mathbb{R}^{d}$ is an unbounded set of the form $L \oplus B$ with direction space $L \in \mathbb{G}(d, k)$ (= the Grassmannian of $k$-dimensional linear subspaces of $\mathbb{R}^{d}$ ), $k=1, \ldots, d-1$, and a convex, compact subset $B$ of the orthogonal complement $L^{\perp}$ called base of the cylinder, see e.g. [16], [12], [20] for details. Throughout this paper the orientation of the direction space $L$ is suppressed and the restriction of convexity of $B$ will be dropped. The general notion of a point process of cylinders (briefly cylinder process subsequently abbreviated by CP) has been first considered in [20]. In order to find explicit formulae for numerical characteristics of union sets of CP's such as volume fraction, covariance etc. one needs specific distributional assumptions determining shape, direction and position of the random cylinders. In order to describe various real-life random set structures, it is quite natural to assume that the sizes and the spatial positions of cylinders are governed by an independently marked Poisson process. Following the concept of Poisson processes defined on the space of cylinders with bases in the convex ring, Poisson cylinder processes (briefly PCP's) have been studied in [17] with applications in modelling materials consisting of long thick fibres or thick membranes. 
To be precise in describing our problem, we first introduce some notation and give a rigorous definition of a stationary PCP (which slightly differs from that in [17]). For this, let $\left\{e_{1}, \ldots, e_{d}\right\}$ denote the usual orthonormal basis of $\mathbb{R}^{d}$ defining the orthogonal subspaces $E_{k}=$ $\operatorname{span}\left\{e_{d-k+1}, \ldots, e_{d}\right\}$ and $E_{k}^{\perp}=\operatorname{span}\left\{e_{1}, \ldots, e_{d-k}\right\}$, where $k \in\{1, \ldots, d-1\}$ is fixed in what follows. It is well-known from differential geometry that for any given $L \in \mathbb{G}(d, k)$ there exists an equivalence class $\mathbf{O}_{L} \in \mathbb{S O}_{d} / \mathbb{S}\left(\mathbb{O}_{d-k} \times \mathbb{O}_{k}\right)$ of orthogonal matrices $O \in \mathbb{R}^{d \times d}$ with $\operatorname{det}(O)=1$ such that $O E_{k}=L$. In other words two matrices $O, \widehat{O} \in \mathbb{S O}_{d}$ belong to $\mathbf{O}_{L}$ iff $O E_{k}=\widehat{O} E_{k}=L$ and $O^{-1} \widehat{O} \in \mathbb{S}\left(\mathbb{O}_{d-k} \times \mathbb{O}_{k}\right)$, where $\mathbb{S}\left(\mathbb{O}_{d-k} \times \mathbb{O}_{k}\right)$ coincides with the set

$$
\left\{\left(\begin{array}{cc}
A & 0 \\
0 & B
\end{array}\right): A \in \mathbb{R}^{(d-k) \times(d-k)}, B \in \mathbb{R}^{k \times k}, A^{T}=A^{-1}, B^{T}=B^{-1}, \operatorname{det}(A) \operatorname{det}(B)=1\right\} .
$$

We identify each equivalence class $\mathbf{O}_{L}$ with a single representative $O_{L} \in \mathbf{O}_{L}$ and write somewhat loosely $O_{L} \in \mathbb{S O}_{d} / \mathbb{S}\left(\mathbb{O}_{d-k} \times \mathbb{O}_{k}\right)$. Further, since $\operatorname{dim} \mathbb{G}(d, k)=(d-k) k$, see Chapt. 16.11 in [2], there exists a measurable mapping from a bounded Borel parameter set $\Theta_{d, k} \subset \mathbb{R}^{(d-k) k}$ onto $\left\{O_{L}: L \in \mathbb{G}(d, k)\right\}$. Note that the explicit shape of this mapping seems to be known only for special cases, e.g. for $d=2, k=1$ or $d=3, k=1$

$$
O_{L}(\theta)=\left(\begin{array}{cc}
\cos \theta & -\sin \theta \\
\sin \theta & \cos \theta
\end{array}\right) \quad \text { or } \quad O_{L}\left(\theta_{1}, \theta_{2}\right)=\left(\begin{array}{ccc}
\sin \theta_{1} & \cos \theta_{1} \cos \theta_{2} & \cos \theta_{1} \sin \theta_{2} \\
-\cos \theta_{1} & \sin \theta_{1} \cos \theta_{2} & \sin \theta_{1} \sin \theta_{2} \\
0 & -\sin \theta_{2} & \cos \theta_{2}
\end{array}\right)
$$

for $\theta \in \Theta_{2,1}=[0, \pi)$ resp. $\left(\theta_{1}, \theta_{2}\right) \in \Theta_{3,1}=[0,2 \pi) \times\left[0, \frac{\pi}{2}\right)$.

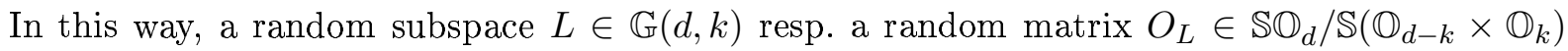
can simply be described by the distribution of a random vector in $\Theta_{d, k}$. Throughout in this paper all random elements are defined on a common probability space $[\Omega, \mathfrak{F}, \mathrm{P}]$ and $\mathrm{E}$ (resp. Var) denotes expectation (resp. variance) w.r.t. P. In particular, let $\left(O_{0}, \Xi_{0}\right)$ be a measurable mapping from $[\Omega, \mathfrak{F}, \mathrm{P}]$ into the product space $\Omega_{d, k}=\mathbb{S O}_{d} / \mathbb{S}\left(\mathbb{O}_{d-k} \times \mathbb{O}_{k}\right) \times \mathcal{K}_{d-k}$, where $\mathcal{K}_{d-k}$ denotes the space of non-void compact subsets of $\mathbb{R}^{d-k}$ equipped with the Hausdorff metric. The image measure $Q:=\mathrm{P} \circ\left(O_{0}, \Xi_{0}\right)^{-1}$ acting on the corresponding Borel product $\sigma$-field $\mathfrak{B}\left(\Omega_{d, k}\right)$ determines the joint distribution of the (not necessarily independent) random elements $O_{0}$ and $\Xi_{0}$. Now we are in a position to introduce a stationary independently marked Poisson process $\Pi_{\lambda, Q}=\sum_{i \geq 1} \delta_{\left[P_{i},\left(O_{i}, \Xi_{i}\right)\right]}$ with intensity $\lambda$ and mark distribution $Q(\cdot)$, i.e. $\Pi_{\lambda, Q}(\cdot)$ is a random locally finite counting measure (shift-invariant in the first component) on the Borel subsets of $\mathbb{R}^{d-k} \times \Omega_{d, k}$ such that the numbers $\Pi_{\lambda, Q}(B \times L)$ are Poisson distributed with mean $\lambda|B|_{d-k} Q(L)$ for any bounded $B \in \mathfrak{B}\left(\mathbb{R}^{d-k}\right)$ (with Lebesgue measure $|\cdot|_{d-k}$ ) and $L \in \mathfrak{B}\left(\Omega_{d, k}\right)$, see [1] for a standard reference on general (Poisson) point processes. This definition implies that the numbers of atoms of the unmarked Poisson process $\Pi_{\lambda}=\sum_{i \geq 1} \delta_{P_{i}}$ located in disjoint subsets of $\mathbb{R}^{d-k}$ are independent and the marks $\left(O_{i}, \Xi_{i}\right)$ associated with the atoms $P_{i}$ are i.i.d. copies of $\left(O_{0}, \Xi_{0}\right) \sim Q$ and independent of $\Pi_{\lambda}$.

Furthermore, we need two important formulae for $\Pi_{\lambda, Q}$, where each of them characterizes the distribution of $\Pi_{\lambda, Q}$ : The probability generating functional $G_{\lambda, Q}[v]=\mathrm{E}\left(\prod_{i \geq 1} v\left(P_{i}, O_{i}, \Xi_{i}\right)\right)$ of $\Pi_{\lambda, Q}$ takes the form 


$$
G_{\lambda, Q}[v]=\exp \left\{-\lambda \int_{\mathbb{R}^{d-k}} \int_{\Omega_{d, k}}(1-v(x, O, K)) Q(\mathrm{~d}(O, K)) \mathrm{d} x\right\}
$$

for any measurable function $v: \mathbb{R}^{d-k} \times \Omega_{d, k} \mapsto[0,1]$ such that $1-v(\cdot, O, K)$ has bounded support for $(O, K) \in \Omega_{d, k}$, whereas the $n$ th-order Campbell formula reads for any $n \in \mathbb{N}$ as follows:

$$
\mathrm{E}\left(\sum_{i_{1}, \ldots, i_{n} \geq 1}^{*} \prod_{j=1}^{n} f_{j}\left(P_{i_{j}}, O_{i_{j}}, \Xi_{i_{j}}\right)\right)=\lambda^{n} \prod_{j=1}^{n} \int_{\mathbb{R}^{d-k}} \int_{\Omega_{d, k}} f_{j}(x, O, K) Q(\mathrm{~d}(O, K)) \mathrm{d} x
$$

for non-negative measurable functions $f_{1}, \ldots, f_{n}: \mathbb{R}^{d-k} \times \Omega_{d, k} \mapsto \mathbb{R}^{1}$, where the sum $\sum^{*}$ on the left-hand side of (1.2) runs over all $n$-tuples of pairwise distinct indices $i_{1}, \ldots, i_{n} \geq 1$, see [1] or [19], [16].

Definition: Given the independently marked Poisson process $\Pi_{\lambda, Q}=\sum_{i \geq 1} \delta_{\left[P_{i},\left(O_{i}, \Xi_{i}\right)\right]}$ satisfying the above assumptions, by a stationary PCP we understand a countable family of cylinders

$$
\Pi_{c y l}^{(d, k)}(\lambda, Q):=\left\{O_{i}\left(\left(\Xi_{i}^{\prime}+P_{i}^{\prime}\right) \oplus E_{k}\right), i \geq 1\right\}=\left\{O_{i}\left(\left(\Xi_{i}+P_{i}\right) \times \mathbb{R}^{k}\right), i \geq 1\right\}
$$

with $\Xi_{i}^{\prime}+P_{i}^{\prime}=\left\{\left(x+P_{i}, 0, \ldots, 0\right): x \in \Xi_{i}\right\} \subset E_{k}^{\perp}$ for $i \geq 1$.

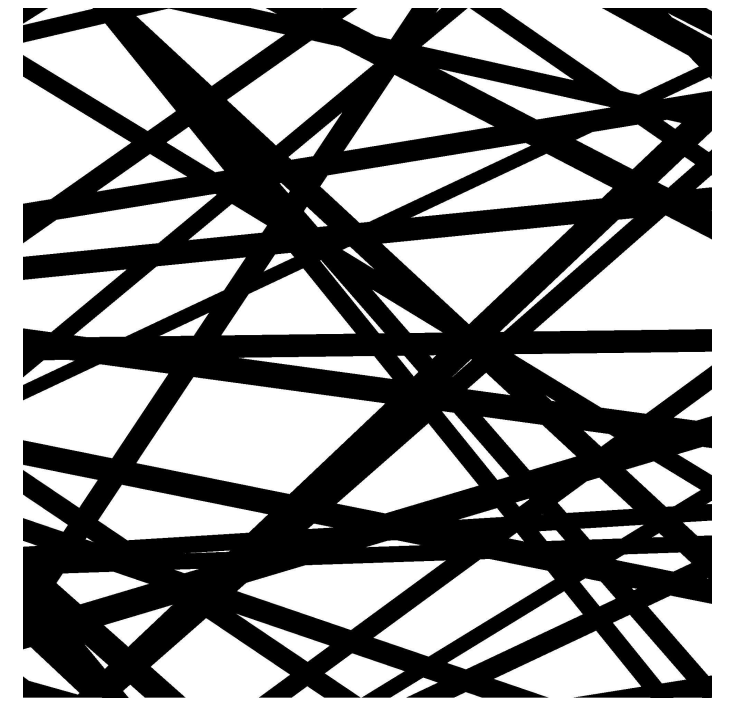

Fig.1 Planar anisotropic PCP in a square

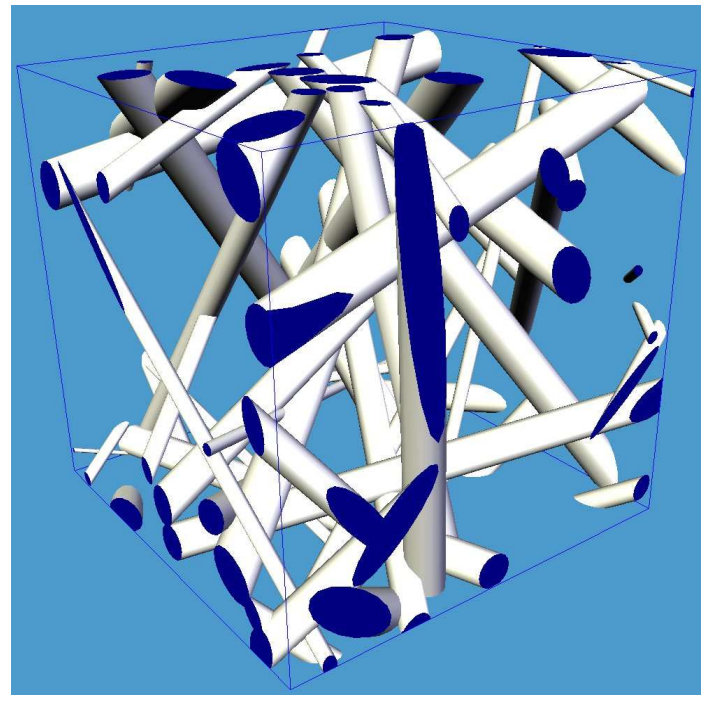

Fig.2 Spatial isotropic PCP in a cube

In this paper we are mainly interested in the random union set

$$
\Xi_{c y l}^{(d, k)}(\lambda, Q)=\bigcup_{i \geq 1} O_{i}\left(\left(\Xi_{i}+P_{i}\right) \times \mathbb{R}^{k}\right)
$$

derived from (1.3) and, in particular, in the asymptotic behaviour (after centering and scaling) of the random $d$-volume $V_{\varrho}^{(d, k)}=\left|\Xi_{c y l}^{(d, k)}(\lambda, Q) \cap \varrho W\right|_{d}$ as $\varrho \rightarrow \infty$ for a fixed set $W \in \mathcal{K}_{d}$ chosen star-shaped (w.r.t. the origin o) such that $b\left(\mathbf{o}, \delta_{W}\right) \subseteq W \subseteq b(\mathbf{o}, 1)$ for some $\delta_{W}>0$. Here $b(x, r)$ is a closed ball in $\mathbb{R}^{d}$ with radius $r \geq 0$ centered at $x \in \mathbb{R}^{d}$. 
Remark 1: In the degenerate case $k=0$ (where $E_{0}=\{\mathbf{o}\}$ and $O_{0}=$ unit matrix) the union set (1.3) coincides with well-studied Boolean (or Poisson grain, Poisson blob, Swiss cheese) model in $\mathbb{R}^{d}$ with typical grain $\Xi_{0}$, see [12], [19].

Remark 2: The union set $\Xi_{c y l}^{(d, k)}(\lambda, Q)$ is (P-a.s.) closed if $\mathrm{E}\left|\Xi_{0} \oplus \pi_{d-k}(b(\mathbf{o}, \varepsilon))\right|_{d-k}<\infty$ for some $\varepsilon>0$, where $\pi_{d-k}(y)$ denotes the projection of the vector $y \in \mathbb{R}^{d}$ on its first $d-k$ components in $\mathbb{R}^{d-k}$. In this case the hitting probability $\mathrm{P}\left(\Xi_{c y l}^{(d, k)}(\lambda, Q) \cap C \neq \emptyset\right)$ can be calculated for any $C \in \mathcal{K}_{d}$ by applying (1.1), see Appendix. A realization of a special union set (1.4) for $d=2, k=1$ and $d=3, k=1$ is shown in Fig. 1 and Fig. 2, respectively.

In the next section we state the announced sharp estimates of the higher-order cumulants $\operatorname{Cum}_{n}\left(V_{\varrho}^{(d, k)}\right)$ of the $d$-volume $V_{\varrho}^{(d, k)}$ under the exponential moment condition

$$
m_{a}=\mathrm{E} \exp \left\{a\left|\Xi_{0}\right|_{d-k}\right\}<\infty \text { for some } a>0 .
$$

This condition is by no means sufficient to imply the closedness of $\Xi_{c y l}^{(d, k)}(\lambda, Q)$ with probability 1. There exist simple counter-examples so that $\mathrm{P}\left(\Xi_{c y l}^{(d, k)}(\lambda, Q)\right.$ is closed $)=0$. For instance, if the typical cylinder base is defined by $\Xi_{0}=\underset{1 \leq i_{1}, \ldots, i_{d-k} \leq N}{\bigcup_{j=1}^{\times}} \underset{j}{\stackrel{d-k}{\times}}\left[i_{j}, i_{j}+\frac{1}{N}\right]$ for some positive random integer $N$ satisfying $\mathrm{E} N=\infty$, then the union set is closed with probability 0 , no matter which distribution $O_{0}$ has.

\section{Main Results}

For notational ease we will mostly use the abbreviation $\Xi$ instead of $\Xi_{c y l}^{(d, k)}(\lambda, Q)$. We first recall the fact that the probability space $[\Omega, \mathfrak{F}, \mathrm{P}]$ on which the marked Poisson process $\Pi_{\lambda, Q}=$ $\sum_{i>1} \delta_{\left[P_{i},\left(O_{i}, \Xi_{i}\right)\right]}$ is defined can be chosen in such a way that the mapping $\mathbb{R}^{d} \times \Omega \ni(x, \omega) \mapsto$ $\mathbb{1}_{\Xi(\omega)}(x) \in\{0,1\}$ is measurable w.r.t. the product- $\sigma$-field $\mathfrak{B}\left(\mathbb{R}^{d}\right) \otimes \mathfrak{F}$, see Appendix in [4]. This enables us to apply Fubini's theorem to the $0-1$-valued random field $\left\{\mathbb{1}_{\Xi}(x), x \in \mathbb{R}^{d}\right\}$, and implies among others that its $n$ th-order mixed moments (also called $n$-point probabilities of $\Xi)$

$$
\mathbb{R}^{d n} \ni\left(x_{1}, \ldots, x_{n}\right) \mapsto p_{\Xi}^{(n)}\left(x_{1}, \ldots, x_{n}\right):=\mathrm{E}\left(\prod_{i=1}^{n} \mathbb{1}_{\Xi}\left(x_{i}\right)\right)=\mathrm{P}\left(x_{1} \in \Xi, \ldots, x_{n} \in \Xi\right)
$$

are $\mathfrak{B}\left(\mathbb{R}^{d n}\right)$-measurable for any $n \in \mathbb{N}$ and $p_{\Xi^{c}}^{(n)}\left(x_{1}, \ldots, x_{n}\right)$ takes on the following explicit form

$$
p_{\Xi^{c}}^{(n)}\left(x_{1}, \ldots, x_{n}\right):=\mathrm{E}\left(\prod_{i=1}^{n}\left(1-\mathbb{1}_{\Xi}\left(x_{i}\right)\right)\right)=\exp \left\{-\lambda \mathrm{E}\left|\bigcup_{i=1}^{n}\left(\Xi_{0}-\pi_{d-k}\left(O_{0}^{T} x_{i}\right)\right)\right|_{d-k}\right\},
$$

see Appendix. Likewise, the $n$ th-order mixed cumulants $c_{\Xi c}^{(n)}\left(x_{1}, \ldots, x_{n}\right)$ of $\left\{1-\mathbb{1}_{\Xi}(x), x \in \mathbb{R}^{d}\right\}$ are Borel measurable functions leading to the following integral representation of the $n$ th-order 
cumulant of $V_{\varrho}^{(d, k)}=|\Xi \cap \varrho W|_{d}$, see Appendix (5.2),

$$
\operatorname{Cum}_{n}\left(V_{\varrho}^{(d, k)}\right)=(-1)^{n} \int_{(\varrho W)^{n}} c_{\Xi^{c}}^{(n)}\left(x_{1}, \ldots, x_{n}\right) \mathrm{d}\left(x_{1}, \cdots, x_{n}\right) \quad \text { for } \quad n \geq 2
$$

We are now in a position to formulate our main results.

Theorem 1. Let $\Xi$ be the union set (1.4) of the stationary $\operatorname{PCP} \Pi_{c y l}^{(d, k)}(\lambda, Q)$ with compact typical cylinder base $\Xi_{0} \subset \mathbb{R}^{d-k}$ satisfying (1.5) and $M_{1}=\mathrm{E}\left|\Xi_{0}\right|_{d-k}>0$. Further, let $W \subset \mathbb{R}^{d}$ be compact and star-shaped w.r.t. o satisfying $b\left(\mathbf{o}, \delta_{W}\right) \subseteq W \subseteq b(\mathbf{o}, 1)$ for some $\delta_{W} \in(0,1)$. Then

$$
\left|\operatorname{Cum}_{n}\left(V_{\varrho}^{(d, k)}\right)\right| \leq \varrho^{(n-1) k+d}(n-1) ! H_{a} \Delta_{a}^{n-2} \quad \text { for } \quad n \geq 2, \varrho>0,
$$

where $H_{a}=2^{2 k+1}|W|_{d} \lambda m_{a}\left(1+\exp \left\{\lambda M_{1}\right\}\right) / a^{2}$ and $\Delta_{a}=2^{2 k+3}\left(a+\lambda m_{a}\right)\left(1+\exp \left\{\lambda M_{1}\right\}\right) / a^{2}$.

The next Theorem 2 states Cramér's large deviations relations for the random $d$-volume $V_{\varrho}^{(d, k)}$ and an optimal Berry-Esseen bound of the distance between the distribution functions

$F_{\varrho}(x)=\mathrm{P}\left(\frac{V_{\varrho}^{(d, k)}-\varrho^{d}|W|_{d}\left(1-\exp \left\{-\lambda \mathrm{E}\left|\Xi_{0}\right|_{d-k}\right\}\right)}{\sigma_{\varrho} \varrho^{(d+k) / 2}} \leq x\right) \quad$ and $\quad \Phi(x)=\frac{1}{\sqrt{2 \pi}} \int_{-\infty}^{x} e^{-t^{2} / 2} \mathrm{~d} t$,

where $\mathrm{P}(\mathbf{o} \in \Xi)=\mathrm{E}\left|\Xi \cap[0,1]^{d}\right|_{d}=1-\exp \left\{-\lambda \mathrm{E}\left|\Xi_{0}\right|_{d-k}\right\}$ is just the volume fraction of the stationary random set (1.4) and the normalized variance $\sigma_{\varrho}^{2}$ of $V_{\varrho}^{(d, k)}$ satisfies the estimate

$$
0<c_{1} \leq \sigma_{\varrho}^{2} \leq c_{2}<\infty \quad \text { for all } \varrho \geq 1 \quad \text { with } \quad \sigma_{\varrho}^{2}=\operatorname{Var}\left(V_{\varrho}^{(d, k)}\right) / \varrho^{d+k}
$$

and $c_{1}, c_{2}$ are constants not depending on $\varrho \geq 1$, see Lemma 1 below.

Theorem 2. Let the assumptions of Theorem 1 be satisfied. Then the following asymptotic relations hold in the interval $0 \leq x \leq \sigma_{\varrho} \varrho^{(d-k) / 2} / 2 \Delta_{a}\left(1+4 H_{a, \varrho}\right)$ with $H_{a, \varrho}=H_{a} / 2 \sigma_{\varrho}^{2}$ :

$$
\frac{1-F_{\varrho}(x)}{1-\Phi(x)}=\exp \left\{\frac{x^{3}}{\sigma_{\varrho} \varrho^{(d+k) / 2}} \sum_{s=0}^{\infty} \mu_{s}^{(\varrho)}\left(\frac{x}{\sigma_{\varrho} \varrho^{(d+k) / 2}}\right)^{s}\right\}\left(1+\mathcal{O}\left(\frac{1+x}{\varrho^{(d-k) / 2}}\right)\right)
$$

and

$$
\frac{F_{\varrho}(-x)}{\Phi(-x)}=\exp \left\{\frac{-x^{3}}{\sigma_{\varrho} \varrho^{(d+k) / 2}} \sum_{s=0}^{\infty} \mu_{s}^{(\varrho)}\left(\frac{-x}{\sigma_{\varrho} \varrho^{(d+k) / 2}}\right)^{s}\right\}\left(1+\mathcal{O}\left(\frac{1+x}{\varrho^{(d-k) / 2}}\right)\right)
$$


as $\varrho \longrightarrow \infty$, where the coefficients $\mu_{s}^{(\varrho)}$ are defined by

$$
\mu_{s}^{(\varrho)}=\frac{1}{(s+2)(s+3)} \sum_{j=1}^{s+1}(-1)^{j-1}\left(\begin{array}{c}
s+j+1 \\
j
\end{array}\right) \sum_{s_{1}+\ldots+s_{j}=s+1}^{>} \prod_{i=1}^{j} \frac{\operatorname{Cum}_{s_{i}+2}\left(V_{\varrho}^{(d, k)}\right)}{\operatorname{Var}\left(V_{\varrho}^{(d, k)}\right)\left(s_{i}+1\right) !}
$$

Here the sum $\sum^{>}$runs over $j$-tuples of positive integers and the series in (2.5) and (2.6) converges absolutely due to the estimate $\left|\mu_{s}^{(\varrho)}\right| \leq 4 H_{a, \varrho} \Delta_{a} \varrho^{k(s+1)}\left(2 \Delta_{a}\left(1+4 H_{a, \varrho}\right)\right)^{s} /(s+2)(s+3)$ for all $s \geq 0$.

Furthermore, there exists some constant $c_{3}>0$ (depending on $a, \lambda, m_{a}$ and $\left.c_{1}, c_{2}\right)$ such that

$$
\sup _{x \in \mathbb{R}^{1}}\left|F_{\varrho}(x)-\Phi(x)\right| \leq c_{3} \varrho^{-(d-k) / 2} \quad \text { for all } \varrho \geq 1 .
$$

Theorem 2 is derived from (2.3) combined with a general lemma on large deviations for a single random variable with mean 0 and variance 1 due to V. Statulevičius [18], see also Lemma 2.3 in the monograph [15]. The relations (2.5) and (2.6) are of particular interest at $x=$ $\varepsilon|W|_{d} \varrho^{(d-k) / 2} / \sigma_{\varrho}$ for small $\varepsilon>0$.

It is an open question whether the Berry-Esseen estimate (2.8) can be obtained under weaker conditions on the cylinder base. Perhaps it suffices to require $\mathrm{E}\left|\Xi_{0}\right|_{d-k}^{3}<\infty$ as one would expect from the CLT for independent random variables. In [8] the authors prove the central limit theorem $F_{\varrho}(x) \underset{\varrho \rightarrow \infty}{\longrightarrow} \Phi(x)$ for $x \in \mathbb{R}^{1}$ under $\mathrm{E}\left|\Xi_{0}\right|_{d-k}^{2}<\infty$ without rates of convergence.

We mention further that the above theorems can be extended to analogous results for estimators of the covariance $C_{\Xi^{c}}(u)=\mathrm{P}\left(\mathbf{o} \in \Xi^{c}, u \in \Xi^{c}\right)$ for fixed $u \in \mathbb{R}^{d}$, see e.g. [19], [12] and [17]. This is seen from the obvious relation $C_{\Xi^{c}}(u)=1-\mathrm{P}(\mathbf{o} \in \Xi \cup(\Xi-u))$ and the fact that the union $\Xi \cup(\Xi-u)$ takes the form (1.4) with typical base $\Xi_{0} \cup\left(\Xi_{0}-\pi_{d-k}\left(O_{0}^{T} u\right)\right)$.

The rest of this paper is organized as follows: In the next Sect. 3, we derive bounds for the variance of the volume $V_{\varrho}^{(d, k)}$ and Sect. 4 contains the rather technical proof of the cumulant estimates (2.3). At the end of Sect. 4 we show how to apply the large deviations lemma in [18] to our situation. In the Appendix we recall some basic facts on mixed moments and cumulants connected with random set (1.4) and the random 0-1-field $\left\{\mathbb{1}_{\Xi}(x), x \in \mathbb{R}^{d}\right\}$. Finally, a criterion for (non-)closedness of $\Xi_{c y l}^{(d, k)}(\lambda, Q)$ is given in analogy to that in [4] for Boolean models.

\section{Lower and Upper Bounds for the Variance}

In this section we derive a lower and an upper bound for the variance of $V_{\varrho}^{(d, k)}=|\Xi \cap \varrho W|_{d}$ provided the second moment of $\left|\Xi_{0}\right|_{d-k}$ exists. For this end we first derive a closed-term expression 
of the variance $\operatorname{Var}\left(|\Xi \cap B|_{d}\right)$ for any bounded Borel set $B \in \mathfrak{B}\left(\mathbb{R}^{d}\right)$ using the above formulae for $p_{\Xi^{c}}^{(2)}(\mathbf{o}, x)$ and $p_{\Xi^{c}}^{(1)}(\mathbf{o})$.

By using the very definition of the one- and two-point probabilities $p_{\Xi}^{(n)}, n=1,2$, and the shiftinvariance and additivity of the Lebesgue measure $|\cdot|_{d-k}$, we deduce from (2.1) that

$$
\begin{aligned}
& p_{\Xi}^{(2)}\left(x_{1}, x_{2}\right)-p_{\Xi}^{(1)}\left(x_{1}\right) p_{\Xi}^{(1)}\left(x_{2}\right)=p_{\Xi^{c}}^{(2)}\left(\mathbf{o}, x_{2}-x_{1}\right)-p_{\Xi^{c}}^{(1)}(\mathbf{o}) p_{\Xi^{c}}^{(1)}(\mathbf{o}) \\
= & \exp \left\{-\lambda \mathrm{E} \mid \Xi_{0} \cup\left(\Xi_{0}-\left.\pi_{d-k}\left(O_{0}^{T}\left(x_{2}-x_{1}\right)\right)\right|_{d-k}\right\}-\exp \left\{-2 \lambda \mathrm{E}\left|\Xi_{0}\right|_{d-k}\right\}\right. \\
= & e^{-2 \lambda M_{1}}\left(\exp \left\{\lambda \mathrm{E} \mid \Xi_{0} \cap\left(\Xi_{0}-\left.\pi_{d-k}\left(O_{0}^{T}\left(x_{2}-x_{1}\right)\right)\right|_{d-k}\right\}-1\right) \quad \text { for } \quad x_{1}, x_{2} \in \mathbb{R}^{d} .\right.
\end{aligned}
$$

Here and below we use the abbreviation $M_{s}=\mathrm{E}\left|\Xi_{0}\right|_{d-k}^{s}$ for $s \in \mathbb{N}$. Hence, by multiple application of Fubini's theorem we get for any bounded $B \in \mathfrak{B}\left(\mathbb{R}^{d}\right)$ that

$$
\begin{aligned}
\operatorname{Var}\left(|\Xi \cap B|_{d}\right) & =\mathrm{E} \int_{B} \int_{B} \mathbb{1}_{\Xi}\left(x_{1}\right) \mathbb{1}_{\Xi}\left(x_{2}\right) \mathrm{d} x_{1} \mathrm{~d} x_{2}-\left(\mathrm{E} \int_{B} \mathbb{1}_{\Xi}(x) \mathrm{d} x\right)^{2} \\
& =\int_{\mathbb{R}^{d}} \int_{\mathbb{R}^{d}} \mathbb{1}_{B}\left(x_{1}\right) \mathbb{1}_{B}\left(x_{2}\right)\left(p_{\Xi}^{(2)}\left(x_{1}, x_{2}\right)-p_{\Xi}^{(1)}\left(x_{1}\right) p_{\Xi}^{(1)}\left(x_{2}\right)\right) \mathrm{d} x_{1} \mathrm{~d} x_{2} \\
& =e^{-2 \lambda M_{1}} \int_{\mathbb{R}^{d}}|B \cap(B-x)|_{d}\left(\exp \left\{\lambda \mathrm{E} \mid \Xi_{0} \cap\left(\Xi_{0}-\left.\pi_{d-k}\left(O_{0}^{T} x\right)\right|_{d-k}\right\}-1\right) \mathrm{d} x .\right.
\end{aligned}
$$

Now we replace $B$ by the star-shaped set $\varrho W$ which increases when $\varrho$ does. In view of the relation $\left\{x \in \mathbb{R}^{d}: \varrho W \cap(\varrho W-x) \neq \emptyset\right\}=\varrho(W \oplus(-W)) \subseteq b(\mathbf{o}, 2 \varrho)$ and the inequality $e^{y}-1 \leq y e^{y}$ for $y \geq 0$ we may write

$$
\begin{aligned}
\operatorname{Var}\left(V_{\varrho}^{(d, k)}\right) & \leq \lambda e^{-\lambda M_{1}}|\varrho W|_{d} \int_{\varrho(W \oplus(-W))} \mathrm{E}\left|\Xi_{0} \cap\left(\Xi_{0}+\pi_{d-k}\left(O_{0}^{T} x\right)\right)\right|_{d-k} \mathrm{~d} x \\
& \leq \lambda|W|_{d} e^{-\lambda M_{1}} \varrho^{d} \mathrm{E} \int_{b(\mathbf{o}, 2 \varrho)}\left|\Xi_{0} \cap\left(\Xi_{0}+\pi_{d-k}(x)\right)\right|_{d-k} \mathrm{~d} x \\
& \left.\left.\leq \lambda|W|_{d} e^{-\lambda M_{1}} \varrho^{d} \mathrm{E} \int_{[-2} \int_{\varrho, 2}\right]^{k} \mathbb{R}^{d-k} \mid \Xi_{0} \cap\left(\Xi_{0}+y_{1}\right)\right)\left.\right|_{d-k} \mathrm{~d} y_{1} \mathrm{~d} y_{2} \\
& =\lambda|W|_{d} e^{-\lambda M_{1}} 4^{k} \mathrm{E}\left|\Xi_{0}\right|_{d-k}^{2} \varrho^{k+d} \\
& \leq \lambda M_{2} e^{-\lambda M_{1}} 2^{d+2 k} \varrho^{k+d} \text { for any } \varrho>0 .
\end{aligned}
$$

To find a positive lower bound of the ratio $\sigma_{\varrho}^{2}$ we make use of $b\left(\mathbf{o}, \delta_{W}\right) \subseteq W$ which implies $\varrho W \cap(\varrho W-x) \supseteq b\left(\mathbf{o}, \delta_{W} \varrho\right) \cap b\left(-x, \delta_{W} \varrho\right)$ and $\varrho(W \oplus(-W)) \subseteq b\left(\mathbf{o}, 2 \delta_{W} \varrho\right)$. This combined 
with $e^{y}-1 \geq y$ for $y \geq 0$ implies

$$
\begin{aligned}
\operatorname{Var}\left(V_{\varrho}^{(d, k)}\right) & \geq \lambda e^{-2 \lambda M_{1}} \int_{b\left(\mathbf{o}, 2 \delta_{W} \varrho\right)}\left|b\left(\mathbf{o}, \delta_{W} \varrho\right) \cap b\left(x, \delta_{W} \varrho\right)\right|_{d} \mathbf{E}\left|\Xi_{0} \cap\left(\Xi_{0}+\pi_{d-k}\left(O_{0}^{T} x\right)\right)\right|_{d-k} \mathrm{~d} x \\
& \geq \lambda e^{-2 \lambda M_{1}} \int_{b\left(\mathbf{o}, \delta_{W} \varrho\right)}\left|b\left(\mathbf{o}, \delta_{W} \varrho\right) \cap b\left(x, \delta_{W} \varrho\right)\right|_{d} \mathbf{E}\left|\Xi_{0} \cap\left(\Xi_{0}+\pi_{d-k}(x)\right)\right|_{d-k} \mathrm{~d} x \\
& \geq \lambda e^{-2 \lambda M_{1}} c(d)\left(\varrho \delta_{W}\right)^{d} \int_{b\left(\mathbf{o}, \delta_{W} \varrho\right)} \mathrm{E}\left|\Xi_{0} \cap\left(\Xi_{0}+\pi_{d-k}(x)\right)\right|_{d-k} \mathrm{~d} x \\
& \geq \lambda e^{-2 \lambda M_{1}} c(d)\left(\varrho \delta_{W}\right)^{d} \int_{\left[-\varrho \delta_{W} / \sqrt{d}, \varrho \delta_{W} / \sqrt{d}\right]^{d}} \mathrm{E}\left|\Xi_{0} \cap\left(\Xi_{0}+\pi_{d-k}(x)\right)\right|_{d-k} \mathrm{~d} x \\
(3.2) & =\lambda 2^{k} d^{-k / 2} e^{-2 \lambda M_{1}}\left(\varrho \delta_{W}\right)^{d+k} c(d) I_{d, k}(\varrho)
\end{aligned}
$$

with

$$
c(d)=\left|b(\mathbf{o}, 1) \cap b\left(e_{1}, 1\right)\right|_{d}>0 \quad \text { and } \quad I_{d, k}(\varrho)=\int_{\left[-\varrho \delta_{W} / \sqrt{d}, \varrho \delta_{W} / \sqrt{d}\right]^{d-k}} \mathrm{E}\left|\Xi_{0} \cap\left(\Xi_{0}+y\right)\right|_{d-k} \mathrm{~d} y .
$$

Making use of $\mathrm{P}\left(\left|\Xi_{0}\right|_{d-k}>0\right)>0$ and standard measure-theoretic arguments it follows that $I_{d, k}(\varrho)>0$ for any $\varrho>0$ and $I_{d, k}(\varrho)$ increases with $\varrho \uparrow \infty$ to the limit $\mathrm{E}\left|\Xi_{0}\right|_{d-k}^{2}$. In this way we confirm the estimate (2.4) with constants

$$
c_{1}=\lambda 2^{k} d^{-k / 2} e^{-2 \lambda M_{1}} \delta_{W}^{d+k} c(d) I_{d, k}(1) \quad \text { and } \quad c_{2}=\lambda 2^{d+2 k} e^{-\lambda M_{1}} M_{2} .
$$

Another consequence of the above estimates is stated in

Lemma 1. Let $\Xi$ be the union set (1.4) of the stationary $\mathrm{PCP} \Pi_{c y l}^{(d, k)}(\lambda, Q)$ with compact cylinder base $\Xi_{0} \subset \mathbb{R}^{d-k}$ satisfying $0<M_{2}=\mathrm{E}\left|\Xi_{0}\right|_{d-k}^{2}<\infty$. Further, let $W \subset R^{d}$ be a compact set satisfying $b\left(\mathbf{o}, \delta_{W}\right) \subseteq W \subseteq b(\mathbf{o}, 1)$ for some $\delta_{W}>0$. Then, we have

$$
\frac{c_{1} M_{2}}{I_{d, k}(1)} \leq \liminf _{\varrho \rightarrow \infty} \frac{\operatorname{Var}\left(|\Xi \cap W \varrho|_{d}\right)}{\varrho^{d+k}} \leq \limsup _{\varrho \rightarrow \infty} \frac{\operatorname{Var}\left(|\Xi \cap W \varrho|_{d}\right)}{\varrho^{d+k}} \leq c_{2}
$$

Remark 3: Lemma 1 reveals that the variance of $V_{\varrho}^{(d, k)}$ grows proportional to the power $|\varrho W|_{d}^{1+k / d}$ of the window volume which expresses long-range dependence within the random set (1.4). The same effect could be observed in investigating the asymptotic behaviour of the total $(d-k)$-volume of intersection $(d-k)$-flats generated by Poisson hyperplane processes in $b(\mathbf{o}, \varrho)$ resp. $\varrho W$ (for convex $W$ ) as $1 \leq \varrho \uparrow \infty$, see [5] resp. [7]. The existence of the limit of the ratio $\sigma_{\varrho}^{2}$ as $\varrho \longrightarrow \infty$ seems to be difficult to prove. So far only in the simplest case $d=2, k=1$ we could give positive answer, see [8]. 


\section{Proofs of the Theorems 1 and 2}

The main part of this section consists of a combination of recursive estimation procedures carried out in several steps which finally result in the estimate (2.3). This proving idea was developed in [4] to obtain a similar estimate for Boolean models. However, the techniques used there had to be extended to unbounded cylinders which cause long-range dependence in contrast to the classical Boolean model. To begin with, using the shift-invariance $c_{\Xi^{c}}^{(n)}\left(x_{1}, \ldots, x_{n}\right)=c_{\Xi^{c}}^{(n)}\left(\mathbf{o}, y_{1}, \ldots, y_{n-1}\right)$ for $y_{i}=x_{i+1}-x_{1}, i=1, \ldots, n-1$, we rewrite (2.2) as follows

$$
\operatorname{Cum}_{n}\left(V_{\varrho}^{(d, k)}\right)=(-1)^{n} \int_{(\varrho(W \oplus(-W)))^{n-1}}\left|\bigcap_{y \in Y_{n-1} \cup\{\mathbf{o}\}}(\varrho W+y)\right|_{d} c_{\Xi^{c}}^{(n)}\left(Y_{n-1} \cup\{\mathbf{o}\}\right) \mathrm{d} Y_{n-1}
$$

for any integer $n \geq 2$. Here and in what follows, we denote by $X_{m}=\left\{x_{1}, \ldots, x_{m}\right\}$ and $Y_{n}=\left\{y_{1}, \ldots, y_{n}\right\}$ (unordered) sets of distinct points $x_{1}, \ldots, x_{m} \in \mathbb{R}^{d}$ and $y_{1}, \ldots, y_{n} \in \mathbb{R}^{d}$, respectively. $|Y|$ gives the number of elements of any finite set $Y \subset \mathbb{R}^{d}$. For notational simplicity put $p(Y)=p_{\Xi^{c}}^{(|Y|)}(Y)$ and $c(Y)=c_{\Xi^{c}}^{(|Y|)}(Y)$ so that, in view of $(2.1)$, we may write

$$
p(Y)=\exp \left\{-\lambda \mathrm{E}\left|\Xi_{0}(Y)\right|_{d-k}\right\} \quad \text { with } \quad \Xi_{0}(Y):=\bigcup_{y \in Y}\left(\Xi_{0}-\pi_{d-k}\left(O_{0}^{T} y\right)\right) .
$$

Further, write $\Xi_{0}^{c}(Y)$ for the complement of $\Xi_{0}(Y)$ in $\mathbb{R}^{d-k}$ and put $\Xi_{0}(\emptyset)=\emptyset, \Xi_{0}^{c}(\emptyset)=$ $\mathbb{R}^{d-k}, p(\emptyset)=1$, and $c(\emptyset)=0$. Note that $c(\{y\})=1-c_{\Xi}^{(1)}(y)=p(\{y\})=\exp \left\{-\lambda M_{1}\right\}$ for any $y \in \mathbb{R}^{d}$. Since $W \oplus(-W) \subseteq b(\mathbf{o}, 2)$ as consequence of $W \subseteq b(\mathbf{o}, 1)$, it follows from (4.1) that

$$
\left.\left.\left|\operatorname{Cum}_{n+1}(\mid \Xi \cap \varrho W)\right|_{d}\right)\left.\left|\leq \varrho^{d}\right| W\right|_{d} \int_{(b(\mathbf{o}, 2 \varrho))^{n}} \mid c\left(\{\mathbf{o}\} \cup Y_{n}\right\}\right) \mid \mathrm{d} Y_{n}
$$

The (mixed) cumulant functions $c(Y)$ are connected with the (mixed) moment functions $p(U)$, $\emptyset \neq U \subseteq Y$, of the random field $\left\{\mathbb{1}_{\Xi^{c}}(x), x \in \mathbb{R}^{d}\right\}$ by

$$
c(Y)=\sum_{j=1}^{|Y|}(-1)^{j-1}(j-1) ! \sum_{U_{1} \cup \cdots \cup U_{j}=Y} p\left(U_{1}\right) \cdots p\left(U_{j}\right) \quad \text { for any finite } \quad Y \subset \mathbb{R}^{d},
$$

where the inner sum runs over all decompositions of $Y$ into pairwise disjoint, non-empty subsets $U_{1}, \ldots, U_{j}$. This formula follows directly by calculating the derivatives in (5.1). The equivalent relationships $c(Y)=p(Y)-\sum_{\emptyset \subset X \subset Y} c(X) p(Y \backslash X)$ or

$$
c\left(\{x\} \cup Y_{n}\right)=p\left(\{x\} \cup Y_{n}\right)-\sum_{\emptyset \subseteq Y \subset Y_{n}} c(\{x\} \cup Y) p\left(Y_{n} \backslash Y\right) \quad \text { for } \quad x \in \mathbb{R}^{d} \backslash Y_{n}
$$

do not really help to establish sharp upper bounds of the integral on the rhs of (4.3). Rather 
than this we introduce the more general functions $X_{m} \times Y_{n} \mapsto c\left(X_{m}, Y_{n}\right)$ for arbitrary $m \geq 1$ and $n \geq 1$ (with $X_{m} \cap Y_{n}=\emptyset$ ) by using the recursive relation

$$
p\left(X_{m} \cup Y_{n}\right)=\sum_{\emptyset \subseteq Y \subseteq Y_{n}} c\left(X_{m}, Y\right) p\left(Y_{n} \backslash Y\right) \quad \text { with } \quad c\left(X_{m}, \emptyset\right)=p\left(X_{m}\right) .
$$

Obviously, $c\left(X_{m}, Y_{n}\right)$ is symmetric in $x_{1}, \ldots, x_{m}$ as well as in $y_{1}, \ldots, y_{n}$, but the $x_{i}$ 's and the $y_{j}$ 's cannot be interchanged. Furthermore, we have $c\left(\{x\}, Y_{n}\right)=c\left(\{x\} \cup Y_{n}\right)$ for $x \notin Y_{n}$ and $n \geq 0$.

As an immediate consequence of (4.4) the recursive relation

$$
c\left(X_{m}, Y_{n}\right)=p\left(X_{m} \cup Y_{n}\right)-\sum_{\emptyset \subseteq Y \subset Y_{n}} c\left(X_{m}, Y\right) p\left(Y_{n} \backslash Y\right)
$$

reveals that $c\left(X_{m}, Y_{n}\right)$ coincides with the $(n+1)$ st-order mixed cumulant of the 0 - 1 -valued random variables $\prod_{i=1}^{m} \mathbb{1}_{\Xi^{c}}\left(x_{i}\right)$ and $\mathbb{1}_{\Xi^{c}}\left(y_{j}\right), j=1, \ldots, n$, that means, formally written that $c\left(X_{m}, Y_{n}\right)=\operatorname{Cum}_{n+1}\left(\mathbb{1}\left\{\Xi \cap X_{m}=\emptyset\right\}, \mathbb{1}_{\Xi^{c}}\left(y_{1}\right), \ldots, \mathbb{1}_{\Xi^{c}}\left(y_{n}\right)\right)$.

The relation

$$
c\left(X_{m}, Y_{n}\right)=\sum_{\emptyset \subseteq Y \subseteq Y_{n}}(-1)^{|Y|} K\left(X_{m}, Y\right) c\left(X_{m-1} \cup Y, Y_{n} \backslash Y\right) \text { for } m+n \geq 1,
$$

where $K(\emptyset, Y)=0$ for $Y \neq \emptyset$ and

$$
K\left(X_{m}, Y\right)=\sum_{\emptyset \subseteq V \subseteq Y}(-1)^{|V|} \frac{p\left(X_{m} \cup V\right)}{p\left(X_{m-1} \cup V\right)} \quad \text { for } \quad m, n \geq 1, \emptyset \subseteq Y \subseteq Y_{n}
$$

has been shown in [4] by direct computation applying Möbius' inversion formula. Setting

$$
p(V \mid U):=\frac{p(U \cup V)}{p(U)}=\mathrm{P}(\Xi \cap V=\emptyset \mid \Xi \cap U=\emptyset),
$$

we can rewrite (4.5) in following way:

$$
c\left(X_{m}, Y_{n}\right)=\frac{p\left(X_{m}\right)}{p\left(X_{m-1}\right)} \sum_{\emptyset \subseteq Y \subseteq Y_{n}}(-1)^{|Y|} S\left(X_{m}, Y\right) c\left(X_{m-1} \cup Y, Y_{n} \backslash Y\right),
$$

where $S(\emptyset, Y)=0$ for $Y \neq \emptyset$ and

$$
S\left(X_{m}, Y\right):=\sum_{\emptyset \subseteq V \subseteq Y}(-1)^{|V|} \frac{p\left(V \mid X_{m}\right)}{p\left(V \mid X_{m-1}\right)} \quad \text { for } \quad \emptyset \subseteq Y \subseteq Y_{n} \text { and } m, n \geq 1
$$

For our random set model (1.4) we get with (4.2) that

$$
\begin{aligned}
\frac{p\left(X_{m} \cup V\right)}{p\left(X_{m-1} \cup V\right)} & =\exp \left\{-\lambda \mathrm{E}\left|\Xi_{0}\right|_{d-k}+\lambda \mathrm{E}\left|\left(\Xi_{0}-\pi_{d-k}\left(O_{0}^{T} x_{m}\right)\right) \cap \Xi_{0}\left(V \cup X_{m-1}\right)\right|_{d-k}\right\} \\
& =\exp \left\{-\lambda \mathrm{E}\left|\left(\Xi_{0}-\pi_{d-k}\left(O_{0}^{T} x_{m}\right)\right) \cap \Xi_{0}^{c}\left(X_{m-1}\right)\right|_{d-k}\right\} \exp \left\{E\left(X_{m}, V\right)\right\}
\end{aligned}
$$


where

$$
E\left(X_{m}, V\right):=\lambda \mathrm{E}\left|\left(\Xi_{0}-\pi_{d-k}\left(O_{0}^{T} x_{m}\right)\right) \cap \Xi_{0}^{c}\left(X_{m-1}\right) \cap \Xi_{0}(V)\right|_{d-k} \text { for } \quad \emptyset \subset V \subseteq Y_{n}
$$

and $E\left(X_{m}, \emptyset\right)=0$.

This leads to $p\left(V \mid X_{m}\right) / p\left(V \mid X_{m-1}\right)=\exp \left\{E\left(X_{m}, V\right)\right\}$ and thus

$$
S\left(X_{m}, Y\right)=\sum_{\emptyset \subseteq V \subseteq Y}(-1)^{|V|} \exp \left\{E\left(X_{m}, V\right)\right\} \quad \text { for } \quad Y \subseteq Y_{n}
$$

and $S\left(X_{m}, \emptyset\right)=1$ since $E\left(X_{m}, \emptyset\right)=0$.

As a simple consequence of (4.6) and $c\left(X_{m}, \emptyset\right)=p\left(X_{m}\right) \leq p\left(X_{m-1}\right) \leq 1$ we get the inequality

$$
\begin{aligned}
& \int_{(b(\mathbf{o}, 2 \varrho))^{n}}\left|c\left(X_{m}, Y_{n}\right)\right| \mathrm{d} Y_{n} \leq \int_{(b(\mathbf{o}, 2 \varrho))^{n}}\left|c\left(X_{m-1}, Y_{n}\right)\right| \mathrm{d} Y_{n}+\int_{(b(\mathbf{o}, 2 \varrho))^{n}}\left|S\left(X_{m}, Y_{n}\right)\right| \mathrm{d} Y_{n} \\
+ & \sum_{\emptyset \subset Y \subset Y_{n}} \int_{(b(\mathbf{o}, 2 \varrho))^{|Y|}}\left|S\left(X_{m}, Y\right)\right| \mathrm{d} Y \sup _{Y} \int_{(b(\mathbf{o}, 2 \varrho))^{n-|Y|}}\left|c\left(X_{m-1} \cup Y, Y_{n} \backslash Y\right)\right| \mathrm{d}\left(Y_{n} \backslash Y\right) .
\end{aligned}
$$

For any $m \geq 1$ we have $c\left(X_{m},\{y\}\right)=p\left(X_{m} \cup\{y\}\right)-p\left(X_{m}\right) p(\{y\})(\geq 0)$ and thus, by (4.2),

$$
\begin{aligned}
& c\left(X_{m},\{y\}\right)=\exp \left\{-\lambda \mathrm{E}\left|\Xi_{0}\left(X_{m} \cup\{y\}\right)\right|_{d-k}\right\}-\exp \left\{-\lambda \mathrm{E}\left|\Xi_{0}\left(X_{m}\right)\right|_{d-k}-\lambda \mathrm{E}\left|\Xi_{0}\right|_{d-k}\right\} \\
= & \exp \left\{-\lambda \mathrm{E}\left|\Xi_{0}\left(X_{m} \cup\{y\}\right)\right|_{d-k}\right\}\left(1-\exp \left\{-\lambda \mathrm{E}\left|\Xi_{0}\left(X_{m}\right) \cap\left(\Xi_{0}-\pi_{d-k}\left(O_{0}^{T} y\right)\right)\right|_{d-k}\right\}\right) \\
\leq & \lambda \exp \left\{-\lambda \mathrm{E}\left|\Xi_{0}\left(X_{m}\right)\right|_{d-k}\right\} \sum_{i=1}^{m} \mathrm{E}\left|\left(\Xi_{0}-\pi_{d-k}\left(O_{0}^{T} x_{i}\right)\right) \cap\left(\Xi_{0}-\pi_{d-k}\left(O_{0}^{T} y\right)\right)\right|_{d-k} .
\end{aligned}
$$

Therefore, since $M_{1}=\mathrm{E}\left|\Xi_{0}\right|_{d-k} \leq \mathrm{E}\left|\Xi_{0}\left(X_{m}\right)\right|_{d-k}$, we get

$$
\int_{b(\mathbf{o}, 2 \varrho)} c\left(X_{m},\{y\}\right) \mathrm{d} y \leq \lambda \exp \left\{-\lambda M_{1}\right\} \sum_{i=1}^{m} \int_{b(\mathbf{o}, 2 \varrho)} \mathrm{E}\left|\Xi_{0} \cap\left(\Xi_{0}-\pi_{d-k}\left(O_{0}^{T}\left(y-x_{i}\right)\right)\right)\right|_{d-k} \mathrm{~d} y .
$$

The integrals on the rhs can be bounded from above uniformly in the $x_{i}$ 's. Multiple application of Fubini's theorem combined with the shift-invariance of the Lebesgue measure in $\mathbb{R}^{d-k}$ yields

$$
\begin{aligned}
\int_{b(\mathbf{o}, 2 \varrho)} \mid \Xi_{0} & \left.\cap\left(\Xi_{0}-\pi_{d-k}\left(O_{0}^{T}(y-x)\right)\right)\right|_{d-k} \mathrm{~d} y=\int_{b(\mathbf{o}, 2 \varrho)}\left|\Xi_{0} \cap\left(\Xi_{0}-\pi_{d-k}(y)+\pi_{d-k}\left(O_{0}^{T} x\right)\right)\right|_{d-k} \mathrm{~d} y \\
& \leq \int_{[-2 \varrho, 2 \varrho]^{k}} \int_{\mathbb{R}^{d-k}}\left|\Xi_{0} \cap\left(\Xi_{0}-z_{1}+\pi_{d-k}\left(O_{0}^{T} x\right)\right)\right|_{d-k} \mathrm{~d} z_{1} \mathrm{~d} z_{2}=(4 \varrho)^{k}\left|\Xi_{0}\right|_{d-k}^{2} .
\end{aligned}
$$


Hence,

$$
\sup _{x \in \mathbb{R}^{d}} \int_{b(\mathbf{o}, 2 \varrho)} \mathrm{E}\left|\left(\Xi_{0}-\pi_{d-k}\left(O_{0}^{T} x\right)\right) \cap\left(\Xi_{0}-\pi_{d-k}\left(O_{0}^{T} y\right)\right)\right|_{d-k} \mathrm{~d} y \leq(4 \varrho)^{k} \mathrm{E}\left|\Xi_{0}\right|_{d-k}^{2}
$$

so that we arrive at the uniform estimate

$$
\sup _{X_{m}} \int_{b(\mathbf{o}, 2 \varrho)} c\left(X_{m},\{y\}\right) \mathrm{d} y \leq C_{m, 1} \varrho^{k} \quad \text { with } \quad C_{m, 1}=4^{k} m \lambda e^{-\lambda M_{1}} M_{2} .
$$

Let us introduce a further non-negative function $T\left(y_{n} ; X_{m}, Y\right)$ by

$$
T\left(y_{n} ; X_{m}, Y\right):=\sum_{\emptyset \subseteq V \subseteq Y}(-1)^{|V|} \exp \left\{-E\left(y_{n} ; X_{m}, V\right)\right\} \quad \text { for } \quad Y \subseteq Y_{n-1}, n \geq 2,
$$

where, for $\emptyset \subseteq V \subseteq Y_{n-1}$,

$E\left(y_{n} ; X_{m}, V\right):=\lambda \mathrm{E}\left|\left(\Xi_{0}-\pi_{d-k}\left(O_{0}^{T} x_{m}\right)\right) \cap \Xi_{0}^{c}\left(X_{m-1}\right) \cap\left(\Xi_{0}-\pi_{d-k}\left(O_{0}^{T} y_{n}\right)\right) \cap \Xi_{0}(V)\right|_{d-k}$.

In the next step of our estimation procedure we determine constants $A_{n}$ and $B_{n}$ only depending on $n, \lambda$, and the first $n+1$ moments $M_{1}, \ldots, M_{n+1}$ of $\left|\Xi_{0}\right|_{d-k}$ such that the uniform estimates

$$
\int_{(b(\mathbf{o}, 2 \varrho))^{n}}\left|S\left(X_{m}, Y_{n}\right)\right| \mathrm{d} Y_{n} \leq A_{n} \varrho^{k n} \text { and } \int_{(b(\mathbf{o}, 2 \varrho))^{n}} T\left(y_{n} ; X_{m}, Y_{n-1}\right) \mathrm{d} Y_{n} \leq B_{n} \varrho^{k n}
$$

hold. The following relations between $S$ - and $T$-functions can be shown in quite analogy to the proof of a corresponding Lemma 4 in [4] :

Lemma 2. For any $m, n \geq 1$ we have

$$
\begin{aligned}
S\left(X_{m}, Y_{n}\right) & =S\left(X_{m}, Y_{n-1}\right)\left(1-\exp \left\{E\left(X_{m},\left\{y_{n}\right\}\right)\right\}\right)-\exp \left\{E\left(X_{m},\left\{y_{n}\right\}\right)\right\} \\
& \times \sum_{\emptyset \subset Y \subseteq Y_{n-1}} T\left(y_{n} ; X_{m}, Y\right) \exp \left\{E\left(X_{m}, Y\right)\right\} S\left(X_{m} \cup Y, Y_{n-1} \backslash Y\right) .
\end{aligned}
$$

Combining the inequality $E\left(X_{m}, Y\right) \leq \lambda \mathrm{E}\left|\left(\Xi_{0}-\pi_{d-k}\left(O_{0}^{T} x_{m}\right)\right) \cap \Xi_{0}(Y)\right|_{d-k} \leq \lambda M_{1}$ and (4.8) leads to

$$
\int_{b(\mathbf{o}, 2 \varrho)}\left|S\left(X_{m},\{y\}\right)\right| \mathrm{d} y=\int_{b(\mathbf{o}, 2 \varrho)}\left(\exp \left\{E\left(X_{m},\{y\}\right)\right\}-1\right) \mathrm{d} y \leq 4^{k} \lambda e^{\lambda M_{1}} M_{2} \varrho^{k} .
$$


Thus, from Lemma 2 and $S\left(X_{m}, \emptyset\right)=1$, it follows after obvious arrangements that

$$
\int_{(b(\mathbf{o}, 2 \varrho))^{n}}\left|S\left(X_{m}, Y_{n}\right)\right| \mathrm{d} Y_{n} \leq 4^{k} \lambda e^{\lambda M_{1}} M_{2} \varrho^{k} \int_{(b(\mathbf{o}, 2 \varrho))^{n-1}}\left|S\left(X_{m}, Y_{n-1}\right)\right| \mathrm{d} Y_{n-1}
$$

$$
+e^{2 \lambda M_{1}} \sum_{j=1}^{n-2}\left(\begin{array}{c}
n-1 \\
j
\end{array}\right) \int_{(b(\mathbf{o}, 2 \varrho))^{j+1}} T\left(y_{j+1} ; X_{m}, Y_{j}\right) \mathrm{d} Y_{j+1}
$$

$$
\times \sup _{Y_{i}} \int_{(b(\mathbf{o}, 2 \varrho))^{n-j-1}}\left|S\left(X_{m} \cup Y_{j}, Y_{n-1} \backslash Y_{j}\right)\right| \mathrm{d}\left(Y_{n-1} \backslash Y_{j}\right)+e^{2 \lambda M_{1}} \int_{(b(\mathbf{o}, 2 \varrho))^{n}} T\left(y_{n} ; X_{m}, Y_{n-1}\right) \mathrm{d} Y_{n}
$$

To make the previous estimate explicit we need upper bounds for the integrals over $T\left(y_{n} ; X_{m}, Y_{n-1}\right)$ w.r.t. the variables $Y_{n}=\left\{y_{1}, \ldots, y_{n-1}, y_{n}\right\}$ for each $n \geq 2$.

Lemma 3. For fixed $n \geq 2$ assume that $M_{n+1}<\infty$. Then, for any $m \geq 1$, both estimates in (4.10) hold with

$$
B_{n}=4^{k n}(n-1) ! \sum_{j=1}^{n-1} \frac{\lambda^{j}}{j !} \sum_{n_{1}+\cdots+n_{j}=n-1}^{>} \frac{M_{n_{1}+2}}{n_{1} !} \prod_{i=2}^{j} \frac{M_{n_{i}+1}}{n_{i} !}
$$

and

$$
A_{n}=A_{n-1} A_{1}+e^{2 \lambda M_{1}} \sum_{j=0}^{n-2}\left(\begin{array}{c}
n-1 \\
j
\end{array}\right) A_{j} B_{n-j} \quad, \quad A_{0}=1, A_{1}=4^{k} \lambda e^{\lambda M_{1}} M_{2} .
$$

Proof of Lemma 3. Let the finite point sets $X_{m}, Y \subseteq Y_{n-1}=\left\{y_{1}, \ldots, y_{n-1}\right\}$ and $y_{n} \in \mathbb{R}^{d}$ be fixed. Using the independently marked Poisson process $\Pi_{\lambda, Q}$ with typical mark $\left(O_{0}, \Xi_{0}\right) \sim Q$ we introduce, in accordance with (1.3) and (1.4), a new stationary PCP and the corresponding stationary random union set $\Xi\left(y_{n} ; X_{m}, Y\right)$ with typical cylinder base $\Xi_{0}\left(y_{n} ; X_{m}, Y\right)=\left(\Xi_{0}-\pi_{d-k}\left(O_{0}^{T} x_{m}\right)\right) \cap \Xi_{0}^{c}\left(X_{m-1}\right) \cap\left(\Xi_{0}-\pi_{d-k}\left(O_{0}^{T} y_{n}\right)\right) \cap \Xi_{0}(Y)$ as follows:

$$
\Xi\left(y_{n} ; X_{m}, Y\right)=\bigcup_{i \geq 1} O_{i}\left(\left(\Xi_{i}\left(y_{n} ; X_{m}, Y\right)+P_{i}\right) \times \mathbb{R}^{k}\right)=\bigcup_{y \in Y} \Xi\left(y_{n} ; X_{m},\{y\}\right),
$$

where $\Xi_{i}\left(y_{n} ; X_{m}, Y\right)=\left(\Xi_{i}-\pi_{d-k}\left(O_{i}^{T} x_{m}\right)\right) \cap \Xi_{i}^{c}\left(X_{m-1}\right) \cap\left(\Xi_{i}-\pi_{d-k}\left(O_{i}^{T} y_{n}\right)\right) \cap \Xi_{i}(Y), i \geq 1$, are i.i.d. random compact sets in $\mathbb{R}^{d-k}$ with $\Xi_{i}(Y)=\bigcup_{y \in Y}\left(\Xi_{i}-\pi_{d-k}\left(O_{i}^{T} y\right)\right)$, see also (4.2).

We first show that $T\left(y_{n} ; X_{m}, Y_{n-1}\right)$ gives just the probability that the origin o lies in each of the union set $\Xi\left(y_{n} ; X_{m},\left\{y_{j}\right\}\right), j=1, \ldots, n-1$. With the above-introduced notation it is rapidly seen that

$$
\mathrm{P}\left(\mathbf{o} \notin \Xi\left(y_{n} ; X_{m}, Y\right)\right)=\exp \left\{-\lambda \mathrm{E}\left|\Xi_{0}\left(y_{n} ; X_{m}, Y\right)\right|_{d-k}\right\}=\exp \left\{-E\left(y_{n} ; X_{m}, Y\right)\right\} .
$$


Taking into account the relations $\sum_{\emptyset \subseteq Y \subseteq Y_{n-1}}(-1)^{|Y|}=0$ and $\Xi\left(y_{n} ; X_{m}, \emptyset\right)=\emptyset$ combined with the second part of (4.15) we find by applying the inclusion-exclusion principle that

$$
\begin{aligned}
T\left(y_{n} ; X_{m}, Y_{n-1}\right) & =\sum_{\emptyset \subseteq Y \subseteq Y_{n-1}}(-1)^{|Y|} \mathrm{P}\left(\mathbf{o} \notin \Xi\left(y_{n} ; X_{m}, Y\right)\right) \\
& =\sum_{\emptyset \subset Y \subseteq Y_{n-1}}(-1)^{|Y|-1} \mathrm{P}\left(\bigcup_{y \in Y}\left\{\mathbf{o} \in \Xi\left(y_{n} ; X_{m},\{y\}\right)\right\}\right) \\
& =\mathrm{P}\left(\bigcap_{j=1}^{n-1}\left\{\mathbf{o} \in \Xi\left(y_{n} ; X_{m},\left\{y_{j}\right\}\right)\right\}\right)=\mathrm{E}\left(\prod_{j=1}^{n-1} \mathbb{1}_{\Xi\left(y_{n} ; X_{m},\left\{y_{j}\right\}\right)}(\mathbf{o})\right),
\end{aligned}
$$

whence, again by Fubini's theorem, it follows that

$$
\int_{(b(\mathbf{o}, 2 \varrho))^{n-1}} T\left(y_{n} ; X_{m}, Y_{n-1}\right) \mathrm{d} Y_{n-1}=\mathrm{E}\left(\int_{b(\mathbf{o}, 2 \varrho)} \mathbb{1}_{\Xi\left(y_{n} ; X_{m},\{y\}\right)}(\mathbf{o}) \mathrm{d} y\right)^{n-1}
$$

Furthermore, the subadditivity of the Dirac measure $\mathbb{1}_{(\cdot)}(\mathbf{o})$ combined with the inclusion relation $\Xi_{i}\left(y_{n} ; X_{m},\{y\}\right) \subseteq\left(\Xi_{i}-\pi_{d-k}\left(O_{i}^{T} x_{m}\right)\right) \cap\left(\Xi_{i}-\pi_{d-k}\left(O_{i}^{T} y_{n}\right)\right) \cap\left(\Xi_{i}-\pi_{d-k}\left(O_{i}^{T} y\right)\right)$ shows that

$$
\begin{aligned}
\int_{b(\mathbf{o}, 2 \varrho)} \mathbb{1}_{\Xi\left(y_{n} ; X_{m},\{y\}\right)}(\mathbf{o}) \mathrm{d} y & \leq \sum_{i \geq 1} \int_{b(\mathbf{o}, 2 \varrho)} \mathbb{1}_{\left(\Xi_{i}\left(y_{n} ; X_{m},\{y\}\right)+P_{i}\right) \times \mathbb{R}^{k}}(\mathbf{o}) \mathrm{d} y \\
& \leq(4 \varrho)^{k} \sum_{i \geq 1} \mathbb{1}_{\left(\Xi_{i}-\pi_{d-k}\left(O_{i}^{T} x_{m}\right)\right) \cap\left(\Xi_{i}-\pi_{d-k}\left(O_{i}^{T} y_{n}\right)\right)}\left(-P_{i}\right)\left|\Xi_{i}\right|_{d-k}
\end{aligned}
$$

In the last line we have replaced the integral of $\mathbb{1}_{\Xi_{i}+P_{i}}\left(\pi_{d-k}\left(O_{i}^{T} y\right)\right)$ over the ball $b(\mathbf{o}, 2 \varrho)$ by the larger term $(4 \varrho)^{k}\left|\Xi_{i}\right|_{d-k}$. Some elementary algebraic rearrangements and the application of the higher-order Campbell's formula (1.2) together with the reflection invariance of stationary Poisson processes enable us to rewrite the $(n-1)$ st moment of the random sum

$$
Z\left(x_{m}, y_{n}\right)=\sum_{i \geq 1} \mathbb{1}_{\left(\Xi_{i}-\pi_{d-k}\left(O_{i}^{T} x_{m}\right)\right) \cap\left(\Xi_{i}-\pi_{d-k}\left(O_{i}^{T} y_{n}\right)\right)}\left(-P_{i}\right)\left|\Xi_{i}\right|_{d-k}
$$

in the following way:

$$
\begin{aligned}
& \sum_{j=1}^{n-1} \sum_{n_{1}+\cdots+n_{j}=n-1}^{>} \frac{(n-1) !}{j ! n_{1} ! \cdots n_{j} !} \mathrm{E} \sum_{i_{1}, \ldots, i_{j} \geq 1}^{*} \prod_{q=1}^{j}\left(\mathbb{1}_{\left(\Xi_{i_{q}}-\pi_{d-k}\left(O_{i_{q}}^{T} x_{m}\right)\right) \cap\left(\Xi_{\left.i_{q}-\pi_{d-k}\left(O_{i_{q}}^{T} y_{n}\right)\right)}\left(P_{i_{q}}\right)\left|\Xi_{i_{q}}\right|_{d-k}^{n_{q}}\right)}\right. \\
= & \sum_{j=1}^{n-1} \sum_{n_{1}+\cdots+n_{j}=n-1}^{>} \frac{\lambda^{j}(n-1) !}{j ! n_{1} ! \cdots n_{j} !} \prod_{q=1}^{j} \mathrm{E}\left(\left|\left(\Xi_{0}-\pi_{d-k}\left(O_{0}^{T} x_{m}\right)\right) \cap\left(\Xi_{0}-\pi_{d-k}\left(O_{0}^{T} y_{n}\right)\right)\right|_{d-k}\left|\Xi_{0}\right|_{d-k}^{n_{q}}\right) .
\end{aligned}
$$


Together with

$\int_{b(\mathbf{o}, 2 \varrho)} \mathrm{E}\left(\left|\left(\Xi_{0}-\pi_{d-k}\left(O_{0}^{T} x_{m}\right)\right) \cap\left(\Xi_{0}-\pi_{d-k}\left(O_{0}^{T} y_{n}\right)\right)\right|_{d-k}\left|\Xi_{0}\right|_{d-k}^{n_{1}}\right) \mathrm{d} y_{n} \leq(4 \varrho)^{k} \mathrm{E}\left|\Xi_{0}\right|_{d-k}^{n_{1}+2}$

we arrive at

$$
\int_{(b(\mathbf{o}, 2 \varrho))^{n}} T\left(y_{n} ; X_{m}, Y_{n-1}\right) \mathrm{d} Y_{n} \leq(4 \varrho)^{k(n-1)} \int_{(b(\mathbf{o}, 2 \varrho))} \mathrm{E}\left(Z\left(x_{m}, y_{n}\right)\right)^{n-1} \mathrm{~d} y_{n} \leq B_{n} \varrho^{k n}
$$

with $B_{n}$ as given in (4.13). Hence, the second estimate in (4.10) is proved.

From (4.11) and (4.12) we obtain the first estimate of (4.10) with a recursive relation for the constants $A_{n}$ with $A_{1}=4^{k} \lambda e^{\lambda M_{1}} M_{2}$ and $A_{0}=1$. More precisely,

$\int_{(b(\mathbf{o}, 2 \varrho))^{n}}\left|S\left(X_{m}, Y_{n}\right)\right| \mathrm{d} Y_{n} \leq A_{1} \varrho^{k} A_{n-1} \varrho^{k(n-1)}+e^{2 \lambda M_{1}} \varrho^{k n} \sum_{j=1}^{n-1}\left(\begin{array}{c}n-1 \\ j\end{array}\right) B_{j+1} A_{n-j-1}=A_{n} \varrho^{k n}$,

which gives (4.14). Thus, the proof of Lemma 3 is completed.

We are now in a position to prove the estimate

$$
\sup _{X_{m}} \int_{\left(b(\mathbf{o}, 2 \varrho)^{n}\right.}\left|c\left(X_{m}, Y_{n}\right)\right| \mathrm{d} Y_{n} \leq C_{m, n} \varrho^{k n} \quad \text { for any } \quad m, n \geq 1
$$

where $C_{m, n}$ depends on $m, n, \lambda$, and $M_{1}, \ldots, M_{n+1}$. From (4.9) we already know that (4.16) is true for $n=1$ and any $m \geq 1$. Inserting the first estimate of (4.10) with constants (4.14) on the rhs of (4.7) we get

$$
\int_{(b(\mathbf{o}, 2 \varrho))^{n}}\left|c\left(X_{m}, Y_{n}\right)\right| \mathrm{d} Y_{n} \leq C_{m-1, n} \varrho^{k n}+A_{n} \varrho^{k n}+\sum_{j=1}^{n-1}\left(\begin{array}{l}
n \\
j
\end{array}\right) A_{j} \varrho^{k j} C_{m-1+j, n-j} \varrho^{k(n-j)},
$$

which immediately implies the estimate (4.16) and the double-index recursion formula

$$
C_{m, n}=A_{n}+\sum_{j=1}^{n}\left(\begin{array}{l}
n \\
j
\end{array}\right) A_{n-j} C_{m-1+n-j, j} \quad \text { with } \quad C_{0, n}=0 \quad \text { for } \quad m, n \geq 1
$$

This equation allows to determine successively all constants $C_{m, n}$ starting with $C_{m, 2}$ depending on $A_{1}, A_{2}$ for all $m \geq 1$ and afterwards $C_{m, 3}$ depending on $A_{1}, A_{2}, A_{3}$ for all $m \geq 1$ etc. For 
example, we have $C_{m, 2}=A_{2}+2 A_{1} C_{m, 1}+C_{m-1,2}$ leading to $C_{m, 2}=m A_{2}+m(m+1) A_{1} C_{1,1}$ for $m \geq 1$.

Having in mind the identity $c\left(\{\mathbf{o}\} \cup Y_{n}\right)=c\left(\{\mathbf{o}\}, Y_{n}\right)$ we deduce from (4.3) and (4.16) that

$$
\left.\left|\operatorname{Cum}_{n}(\mid \Xi \cap \varrho W)\right|_{d}\right)\left.|\leq| W\right|_{d} C_{1, n-1} \varrho^{d+k(n-1)},
$$

where $C_{1, n-1}$ depends on $\lambda$ and $M_{1}, \ldots, M_{n}$. In the final step we determine the growth of the constants $C_{1, n-1}$ in dependence on $n \geq 2$ under the assumption (1.5). In this case we have $M_{n} \leq n ! a^{-n} m_{a}$ for $n \in \mathbb{N}$ so that formula (4.13) yields

$$
B_{n} \leq 4^{k n}(n-1) ! \sum_{j=1}^{n-1} \frac{\lambda^{j}}{j !} \frac{m_{a}^{j}}{a^{j+1}} \sum_{n_{1}+\cdots+n_{j}=n-1}^{>}\left(n_{1}+2\right) \prod_{i=1}^{j} \frac{\left(n_{i}+1\right)}{a^{n_{i}}} .
$$

Since $n+1 \leq 2^{n}$ for $n \in \mathbb{N}$, we have

$$
\sum_{n_{1}+\cdots+n_{j}=n-1}^{>}\left(n_{1}+2\right) \prod_{i=1}^{j} \frac{\left(n_{i}+1\right)}{a^{n_{i}}} \leq \frac{n}{a^{n-1}} \sum_{n_{1}+\cdots+n_{j}=n-1}^{>} 2^{n_{1}+1} \prod_{i=2}^{j} 2^{n_{i}}=\frac{n 2^{n}}{a^{n-1}}\left(\begin{array}{c}
n-2 \\
j-1
\end{array}\right),
$$

which in turn gives

$$
B_{n} \leq \frac{2^{n} 4^{k n}}{a^{n}} n ! \sum_{j=1}^{n-1} \frac{\lambda^{j} m_{a}^{j}}{a^{j}}\left(\begin{array}{c}
n-2 \\
j-1
\end{array}\right)=\frac{\lambda m_{a}}{a}\left(\frac{2 \cdot 4^{k}}{a}\right)^{n}\left(1+\frac{\lambda m_{a}}{a}\right)^{n-2} n ! \text { for } n \geq 2 .
$$

In summary, using the abbreviations,

$$
A=\frac{2^{2 k+1}}{a}\left(1+\exp \left\{\lambda \mathrm{E}\left|\Xi_{0}\right|_{d-k}\right\}\right) \text { and } B=\frac{\lambda \mathrm{E} \exp \left\{a\left|\Xi_{0}\right|_{d-k}\right\}}{a}
$$

the positive constants $A_{n}$ and $B_{n}$ in (4.10) satisfy the estimates $A_{1} \leq A B$ and

$$
A_{n} \leq A^{n} B(1+B)^{n-1} n ! \quad \text { and } \quad B_{n} \leq B\left(\frac{2^{2 k+1}}{a}\right)^{n}(1+B)^{n-2} n ! \text { for } n \geq 2
$$

The first relation follows from (4.14) by induction on $n$. In fact, by $M_{2} \leq 2 m_{a} / a^{2}$, we have

$$
A_{1}=4^{k} \lambda e^{\lambda M_{1}} M_{2} \leq 4^{k} \lambda e^{\lambda M_{1}} \frac{2 m_{a}}{a^{2}}=\frac{2^{2 k+1}}{a} e^{\lambda M_{1}} B \leq A B
$$

and, for $n \geq 2$, we combine the recursive relation (4.14) with $A_{0}=1$ and the second (already proved) estimate in (4.19):

$$
\begin{aligned}
A_{n} & =A_{1} A_{n-1}+e^{2 \lambda M_{1}} \sum_{j=0}^{n-2}\left(\begin{array}{c}
n-1 \\
j
\end{array}\right) A_{j} B_{n-j} \\
& \leq A_{1} A_{n-1}+e^{2 \lambda M_{1}} B \sum_{j=0}^{n-2}\left(\begin{array}{c}
n-1 \\
j
\end{array}\right) A_{j}\left(\frac{2^{2 k+1}}{a}\right)^{n-j}(1+B)^{n-j-2}(n-j) !
\end{aligned}
$$


Replacing $A_{j}$ by $A^{j} B(1+B)^{j-1} j$ ! for $j=1, \ldots, n-1$ we find after some elementary calculations the asserted first estimate in (4.19).

In the same way the recursive relation (4.17) suggests an inductive proof of the estimate

$$
C_{m, n} \leq 2^{m-1} 4^{n-1} A^{n} B(1+B)^{n-1} n ! \text { for } n, m \geq 1,
$$

whence with (4.18) it follows the desired estimate (2.3) completing the proof of Theorem 1.

Now, we apply the general lemma on large deviations including an optimal Berry-Esseen bound proved by V. Statulevičius in [18], see also Lemma 2.3 in [15]. This result is formulated for a single random variable $\xi$ satisfying $\mathrm{E} \xi=0, \operatorname{Var}(\xi)=1$ and $\left|\operatorname{Cum}_{n}(\xi)\right| \leq n ! H / \Delta^{n-2}$ for $n \geq 2$ and some $H \geq 1 / 2$ and $\Delta>0$. In our specific situation $\xi$ is chosen to be the standardized $d$-volume $V_{\varrho}^{(d, k)}$, i.e.,

$$
\xi=\frac{V_{\varrho}^{(d, k)}-\mathrm{E} V_{\varrho}^{(d, k)}}{\sqrt{\operatorname{Var}\left(V_{\varrho}^{(d, k)}\right)}}=\frac{V_{\varrho}^{(d, k)}-\varrho^{d}|W|_{d}\left(1-\exp \left\{-\lambda \mathrm{E}\left|\Xi_{0}\right|_{d-k}\right\}\right)}{\sigma_{\varrho} \varrho^{(d+k) / 2}}
$$

with distribution function $F_{\varrho}(x)=\mathrm{P}\left(V_{\varrho}^{(d, k)} \leq x\right)$. Using (2.3) and the notation introduced in Sect. 2 we obtain that

$$
\left|\operatorname{Cum}_{n}(\xi)\right| \leq(n-1) ! \frac{H_{a} \Delta_{a}^{n-2} \varrho^{d+k(n-1)}}{\left(\operatorname{Var}\left(V_{\varrho}^{(d, k)}\right)\right)^{n / 2}} \leq n ! H_{a, \varrho} / \Delta_{a, \varrho}^{n-2},
$$

where $H_{a, \varrho}=H_{a} / 2 \sigma_{\varrho}^{2}(\geq 1 / 2$ by $(2.3)$ for $n=2)$ and $\Delta_{a, \varrho}=\varrho^{(d-k) / 2} \sigma_{\varrho} / \Delta_{a}$.

These estimates and the Lemma in [18], p. 133, imply the asymptotic relations (2.5) and (2.6) as well as the Berry-Esseen bound (2.8) stated in Theorem 2. It should be noted that, according to the general result in [18] or [15], the relations (2.5) and (2.6) hold in a smaller interval $0 \leq x \leq \delta^{*} \Delta_{a, \varrho}$ for $\delta^{*}<\delta(1+\delta) / 2$, where $\delta \in(0,1)$ is uniquely determined by the equation $(1-\delta)^{3}=6 H_{a, \varrho} \delta$ giving $\delta(1+\delta) / 2 \leq 1 / 2\left(1+4 H_{a, \varrho}\right)$. However, a careful check of the original proof reveals that (2.5) and (2.6) remain valid for larger $x$-values because, in contrast to [18], the explicitly known coefficients $(2.7)$ of the Cramér's series $\mu(x):=\sum_{s \geq 0} \mu_{s}^{(\varrho)}\left(x / \sigma_{\varrho} \varrho^{(d+k) / 2}\right)^{s}$ can be estimated directly by means of (2.3). For doing this, we use $(2.3)$ and $\left(\begin{array}{c}s+j+1 \\ j\end{array}\right) \leq 2^{s+j}$ and get for any $s \geq 0$ that

$$
\begin{aligned}
\left|\mu_{s}^{(\varrho)}\right| & \leq \frac{1}{(s+2)(s+3)} \sum_{j=1}^{s+1} 2^{s+j} \sum_{s_{1}+\ldots+s_{j}=s+1}^{>} \prod_{i=1}^{j}\left(\frac{\Delta_{a}^{s_{i}} \varrho^{k s_{i}} H_{a}}{\sigma_{\varrho}^{2}}\right) \\
& =\frac{2^{s} \Delta_{a}^{s+1} \varrho^{k(s+1)}}{(s+2)(s+3)} \sum_{j=1}^{s+1}\left(\begin{array}{c}
s \\
j-1
\end{array}\right)\left(\frac{2 H_{a}}{\sigma_{\varrho}^{2}}\right)^{j}=\frac{4 H_{a, \varrho} \Delta_{a} \varrho^{k}}{(s+2)(s+3)}\left(2 \Delta_{a} \varrho^{k}\left(1+4 H_{a, \varrho}\right)\right)^{s} .
\end{aligned}
$$

Thus, $\mu(x)$ converges absolutely for $|x| \leq \Delta_{a, \varrho} / 2\left(1+4 H_{a, \varrho}\right)$ such that $|\mu(x)| \leq 2 H_{a, \varrho} \Delta_{a} \varrho^{k}$ proving the validity of (2.5) and (2.6) in the desired interval $0 \leq x \leq \Delta_{a, \varrho} / 2\left(1+4 H_{a, \varrho}\right)$ which completes the proof of Theorem 2 . 


\section{Appendix}

In the previous sections we were dealing with the volume fraction of the random set $\Xi_{c y l}^{(d, k)}(\lambda, Q)$ in various Borel sets. For this purpose it suffices to consider the stationary 0 - 1-random field $\left\{\mathbb{1}_{\Xi}(x), x \in \mathbb{R}^{d}\right\}$ the finite-dimensional distributions of which are given by the family of $n$-point probabilities $p_{\Xi}^{(n)}\left(x_{1}, \ldots, x_{n}\right)=\mathrm{P}\left(x_{1} \in \Xi, \ldots, x_{n} \in \Xi\right)$ or by $p_{\Xi^{c}}^{(n)}\left(x_{1}, \ldots, x_{n}\right)=\mathrm{P}\left(x_{1} \notin \Xi, \ldots, x_{n} \notin \Xi\right)$ for $x_{1}, \ldots, x_{n} \in \mathbb{R}^{d}, n \in \mathbb{N}$. In general, these distributions do not describe the properties of a random set $\Xi$ completely, see [13]. In case of the random set (1.4) one can choose the canonical probability space $[\Omega, \mathfrak{F}, \mathrm{P}]$ on which the marked Poisson point process $\Pi_{\lambda, Q}$ (introduced in Sect. 1) is defined so that the mapping $\mathbb{R}^{d} \times \Omega \ni(x, \omega) \mapsto \mathbb{1}_{\Xi(\omega)}(x)$ turns out $\mathfrak{B}\left(\mathbb{R}^{d}\right) \otimes \mathfrak{F}$-measurable. This follows by repeating the arguments (with obvious changes) proving Proposition 1 in [4]. Hence, Fubini's theorem and the probability generating functional (1.1) can be applied to calculate the $n$-point probabilities of the complement $\Xi^{c}$

$p_{\Xi^{c}}^{(n)}\left(x_{1}, \ldots, x_{n}\right)=\mathrm{P}\left(\Xi \cap\left\{x_{1}, \ldots, x_{n}\right\}=\emptyset\right)=\mathrm{E}\left(\prod_{i \geq 1} \mathbb{1}\left\{O_{i}\left(\left(\Xi_{i}+P_{i}\right) \times \mathbb{R}^{k}\right) \cap\left\{x_{1}, \ldots, x_{n}\right\}=\emptyset\right\}\right)$

which immediately shows the validity of (2.1) provided that $\mathrm{E}\left|\Xi_{0}\right|_{d-k}<\infty$. It is a matter of fact that even the boundedness of the random $(d-k)$-volume $\left|\Xi_{0}\right|_{d-k}$ does not imply the closedness of the random set (1.4). In the theory of random closed sets, see [12] or [13], the distribution of $\Xi$ is uniquely determined by its capacity functional $T_{\Xi}(C)=\mathrm{P}(\Xi \cap C \neq \emptyset)$ defined on the family of non-empty compact sets $C \in \mathcal{K}_{d}$. The union set $\Xi=\Xi_{c y l}^{(d, k)}(\lambda, Q)$ is (P-a.s.) closed if any ball in $\mathbb{R}^{d}$ hits at most finitely many cylinders $O_{i}\left(\left(\Xi_{i}+P_{i}\right) \times \mathbb{R}^{k}\right)$ with probability 1 which in turn is equivalent to $\mathrm{E}\left|\Xi_{0} \oplus \pi_{d-k}(b(\mathbf{o}, \varepsilon))\right|_{d-k}<\infty$ for some $\varepsilon>0$, see [3] for general germ-grain models. Under the latter condition the explicit form of $T_{\Xi}(C)$ can be calculated for (1.4). Applying again the probability generating functional (1.1) with $v(x, O, K)=\mathbb{1}\left\{O\left((K+x) \times \mathbb{R}^{k}\right) \cap C=\emptyset\right\}$ we have

$$
\begin{aligned}
1-T_{\Xi}(C)=\mathrm{P}(\Xi \cap C=\emptyset) & =\mathrm{E}\left(\prod_{i \geq 1} \mathbb{1}\left\{O_{i}\left(\left(\Xi_{i}+P_{i}\right) \times \mathbb{R}^{k}\right) \cap C=\emptyset\right\}\right) \\
& =\exp \left\{-\lambda \mathrm{E} \int_{\mathbb{R}^{d-k}} \mathbb{1}\left\{O_{0}\left(\left(\Xi_{0}+x\right) \times \mathbb{R}^{k}\right) \cap C \neq \emptyset\right\} \mathrm{d} x\right\} .
\end{aligned}
$$

Since $O_{0}\left(\left(\Xi_{0}+x\right) \times \mathbb{R}^{k}\right) \cap C \neq \emptyset$ iff $\left(\Xi_{0}+x\right) \cap \pi_{d-k}\left(O_{0}^{T} C\right) \neq \emptyset$ and the latter is equivalent to $x \in \Xi_{0} \oplus\left(-\pi_{d-k}\left(O_{0}^{T} C\right)\right)$, we arrive at

$$
T_{\Xi}(C)=1-\exp \left\{-\lambda \mathrm{E}\left|\Xi_{0} \oplus\left(-\pi_{d-k}\left(O_{0}^{T} C\right)\right)\right|_{d-k}\right\}, \quad \text { see e.g. } \quad[17]
$$

The following lemma which we formulate without proof extends an analogous statement for Boolean models in [4] to unions of PCP's (1.4). This result implies that, under the assumption $\mathrm{E}\left|\Xi_{0}\right|_{d-k}<\infty$, the additional condition $\mathrm{E}\left|\Xi_{0} \oplus \pi_{d-k}(b(\mathbf{o}, \varepsilon))\right|_{d-k}<\infty$ for some $\varepsilon>0$ is not only sufficient, but even necessary for the closedness of the stationary random union set (1.4).

Lemma 4. Let $\Xi_{0}$ be a compact typical cylinder base of the (1.3) satisfying $\mathrm{E}\left|\Xi_{0}\right|_{d-k}<\infty$ and $\mathrm{E}\left|\Xi_{0} \oplus \pi_{d-k}(b(\mathbf{o}, \varepsilon))\right|_{d-k}=\infty$ for any $\varepsilon>0$. Then $\mathrm{P}\left(\Xi_{c y l}^{(d, k)}(\lambda, Q)\right.$ is closed in $\left.\mathbb{R}^{d}\right)=0$. 
The proof of Lemma 4 is quite similar to that in [4] for Boolean models. The necessary changes and extensions are left to the reader.

Next we put together some basic facts on the "method of cumulants". There exists a huge and widely scattered literature in stochastics, see e.g. [11], and statistical physics in connection with cluster expansions, see e.g. [14], in which cumulant techniques are employed to express the weakness of stochastic dependence between temporally (or spatially) distant parts of random processes (or fields). In statistics and probability theory these cumulant estimates are mainly used to prove asymptotic Gaussianity of functionals of random processes (or fields) over expanding domains. For obtaining even rates of convergence in these limit theorems and exact large deviations probabilities based on cumulant estimates the reader is referred to the monograph [15]. Note that in finding optimal rates the corresponding estimation procedures are partly rather lengthy and sophisticated, see [6] for an example.

Let us recall the definition of the mixed cumulant (semi-invariant) Cum $\left(Y_{1}, \ldots, Y_{n}\right)$ of $n$ random variables $Y_{1}, \ldots, Y_{n}$ (all having a finite $n$th moment). Following [10] we define

$$
\operatorname{Cum}\left(Y_{1}, \ldots, Y_{n}\right)=\left.\mathbf{i}^{-n} \frac{\partial^{n}}{\partial s_{1} \ldots \partial s_{n}} \log \mathrm{E} \exp \left\{\mathbf{i} \sum_{j=1}^{n} s_{j} Y_{j}\right\}\right|_{s_{1}=\cdots=s_{n}=0}
$$

and $\operatorname{Cum}_{n}(Y)=\operatorname{Cum}(Y, \ldots, Y)$ (by setting $Y=Y_{1}=\cdots=Y_{n}$ in (5.1)) denotes the usual $n$th cumulant of $Y$. From (5.1) it is easily seen that $\operatorname{Cum}\left(Y_{1}, \ldots, Y_{n}\right)$ is invariant under permutation of the indices $\{1, \ldots, n\}$ and $\operatorname{Cum}(\ldots, a Y+b Z+c, \ldots)=a \operatorname{Cum}(\ldots, Y, \ldots)+b \operatorname{Cum}(\ldots, Z, \ldots)$ in each component for any $a, b, c \in \mathbb{R}^{1}, n \geq 2$.

Let $\left\{\mathbb{1}_{\Xi}(x), x \in \mathbb{R}^{d}\right\}$ be a measurable 0 - 1-random field, for example, when $\Xi$ coincides with (1.4). Obviously, the mixed cumulant function $c_{\Xi}^{(n)}\left(x_{1}, \ldots, x_{n}\right)=\operatorname{Cum}\left(\mathbb{1}_{\Xi}\left(x_{1}\right), \ldots, \mathbb{1}_{\Xi}\left(x_{n}\right)\right)$ equals $(-1)^{n} \operatorname{Cum}\left(\mathbb{1}_{\Xi^{c}}\left(x_{1}\right), \ldots, \mathbb{1}_{\Xi^{c}}\left(x_{n}\right)\right)$. By combining the identities $\left|\Xi \cap B_{i}\right|=\int_{B_{i}} \mathbb{1}_{\Xi}\left(x_{i}\right) \mathrm{d} x_{i}$ for $i=1, \ldots, n$ with the linearity of (5.1) in each component we get

$$
\operatorname{Cum}\left(\left|\Xi \cap B_{1}\right|, \ldots,\left|\Xi \cap B_{n}\right|\right)=(-1)^{n} \int_{B_{1}} \ldots \int_{B_{n}} c_{\Xi^{c}}^{(n)}\left(x_{1}, \ldots, x_{n}\right) \mathrm{d} x_{1} \cdots \mathrm{d} x_{n}
$$

for any bounded $B_{1}, \ldots, B_{n} \in \mathfrak{B}\left(\mathbb{R}^{d}\right)$. By calculating the logarithmic derivatives in (5.1), see e.g. [10], [15] or [6], the cumulant function $c_{\Xi^{c}}^{(n)}\left(x_{1}, \ldots, x_{n}\right)$ can be expressed by the $k$-point probabilities $p_{\Xi^{c}}^{(k)}\left(x_{i_{1}}, \ldots, x_{i_{k}}\right)$ for $1 \leq i_{1}<\cdots<i_{k} \leq n$ and $k=1, \ldots, n$. 


\section{References}

[1] D.J. Daley and D. Vere-Jones, An Introduction to the Theory of Point Processes, Springer, New York, 1988.

[2] J. Dieudonné, Grundzüge der modernen Analysis, Bd. 3, Dt. Verlag der Wiss., Berlin, 1976.

[3] L. Heinrich, On existence and mixing properties of germ-grain models, Statistics 23, 271 $286,1992$.

[4] L. Heinrich, Large deviations of the empirical volume fraction for stationary Poisson grain models, Ann. Appl. Prob. 15, No. 1A, 392 - 420, 2005.

[5] L. Heinrich, H. Schmidt and V. Schmidt, Central limit theorems for Poisson hyperplane tessellations, Ann. Appl. Prob. 16, 919 - 950, 2005.

[6] L. Heinrich, An almost-Markov-type mixing condition and large deviations for Boolean models on the line, Acta. Appl. Math. 96, 247 - 262, 2007.

[7] L. Heinrich, Central limit theorems for motion-invariant Poisson hyperplanes in expanding convex bodies, Rend. Circ. Mat. Palermo, to appear in 2010, see also Preprint Nr. 043/2007, Institut für Mathematik, Universität Augsburg.

[8] L. Heinrich and M. Spiess, Asymptotic normality of the volume covered by Poisson cylinder processes in large domains, unpublished manuscript 2009.

[9] L.M. Hoffmann, Mixed measures of convex cylinders and quermass densities of Boolean models, Acta. Appl. Math. 105, 141 - 156, 2009.

[10] V.P. Leonov and A.N. Shiryaev, On a method of calculating semi-invariants, Theory Probab. Appl. 4, 319 - 329, 1959.

[11] V.P. Leonov, Some Applications of Higher Cumulants in Random Process Theory (in Russian), Nauka, Moscow, 1964.

[12] G. Matheron, Random Sets and Integral Geometry, Wiley \& Sons, New York, 1975.

[13] I. Molchanov, Theory of Random Sets, Springer, London, 2005.

[14] D. Ruelle, Statistical Mechanics: Rigorous Results, 5nd ed., Addison-Wesley, Reading (Mass.), 1989.

[15] L. Saulis and V. Statulevičius, Limit Theorems for Large Deviations, Kluwer Academic Press, Dordrecht, 1991.

[16] R. Schneider and W. Weil, Stochastic and Integral Geometry, Springer, Berlin, 2008. 
[17] M. Spiess and E. Spodarev, Anisotropic Poisson processes of cylinders, submitted 2009.

[18] V.A. Statulevičius, On large deviations, Z. Wahr.-theorie verw. Gebiete 6, 133 - 144, 1966.

[19] D. Stoyan, W.S. Kendall and J. Mecke, Stochastic Geometry and Its Applications, 2nd ed., Wiley \& Sons, Chichester, 1995.

[20] W. Weil, Point processes of cylinders, particles and flats, Acta Appl. Math. 6, 103 - 136, 1987. 